\title{
Hyperthermic Intraperitoneal Chemotherapy - Fading Perspective in the Light of Modern Systemic Chemotherapy?
}

\author{
Pankaj Kumar Garg ${ }^{a} \quad$ Andreas Brandl $^{b} \quad$ Beate Rau $^{b}$

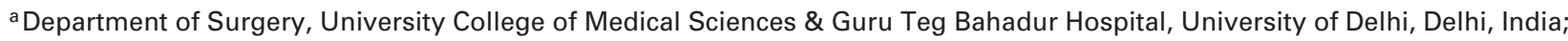 \\ ${ }^{b}$ Department of General Surgery, Campus Virchow Klinikum, Charité - Universitätsmedizin Berlin, Berlin, Germany
}

\section{Keywords}

Cytoreductive surgery - Gastrointestinal cancer .

Hyperthermic intrapertioneal chemotherapy · Peritoneal metastasis - Systemic chemotherapy

\section{Summary}

Background: Peritoneal metastasis (PM) in a gastrointestinal cancer is associated with a poor prognosis. Various guidelines recommend palliative chemotherapy in these patients as cure is not achievable. However, hyperthermic intraperitoneal chemotherapy (HIPEC) in combination with cytoreductive surgery (CRS) can achieve longterm survival with low morbidity in selected patients. During the last decades, CRS and HIPEC have been performed in a number of indications, aiming for long-term survival. What is more, new drugs and treatment regimens demonstrate favorable results. Methods: The recently published important literature on PM in gastrointestinal cancer was analyzed. Results: Resection of isolated PM in a gastrointestinal cancer should be considered, and HIPEC is feasible. However, there is a lack of well-designed randomized controlled trials (RCTs) in gastrointestinal cancer-related PM. Many RCTs are being conducted across the world whose results will be available in 1-2 years. Systemic chemotherapy alone as an adequate management plan for isolated PM in a gastrointestinal cancer is not compatible with a high standard of care. Formulating an optimal plan combining the benefits of CRS and HIPEC coupled with systemic chemother-

Data from this manuscript have in part been presented at the symposium 'Frontiers in Surgical Evolution' on September 21, 2018 in honor of Prof. Dr. Ernst Klar, Rostock, Germany. apy is a necessary task of the multidisciplinary team. Proper selection of the patients for CRS and HIPEC is necessary to achieve maximal oncological benefit and minimal perioperative morbidity and mortality. Conclusions: A multidisciplinary approach combining the benefits of both CRS and HIPEC, and systemic chemotherapy is the way forward to manage those patients with gastrointestinal cancer and PM. Further studies are warranted to identify the selection variables for CRS and HIPEC.

(c) 2018 S. Karger GmbH, Freiburg

\section{Introduction}

Peritoneal metastasis (PM) in any solid cancer has been classically viewed with a great amount of nihilism and as a surrogate marker of imminent cancer-related death. The prognosis of the patients who have gastrointestinal cancer with PM is dismal, and they are usually referred to palliative chemotherapy/best supportive care. There has been a paradigm shift in the intent of management of PM, i.e. from palliative to curative, in a select group of patients in the last two decades, and it has generated a lot of optimism among surgeons and oncologists. Aggressive surgery for PM in a select group of patients with gastrointestinal cancer to achieve almost complete extirpation of the tumor - cytoreductive surgery (CRS) and hyperthermic intraperitoneal chemotherapy (HIPEC), has been shown to be a way forward to achieve good oncological outcomes in a battle almost lost otherwise.

Should we really consider an isolated localized PM as systemic dissemination? The recent trend has been to consider it, at most, a manifestation of regional metastasis that is amenable to locoregional therapy [1]. The last two decades witnessed a number of tri- 
als which addressed localized PM in a gastrointestinal cancer with aggressive surgery and intraperitoneal chemotherapy, and highlighted a significant survival benefit in treated patients.

In the era of modern systemic chemotherapy, the question can be asked about the need for such an aggressive surgical therapy which is potentially morbid. However, the answer lies in the combined approach using the maximal benefit of aggressive surgery and systemic chemotherapy in a multidisciplinary approach as it tends to add up the survival benefit compared to using any modality alone [2-4]. However, it must be emphasized that not all patients with PM are likely to benefit from CRS and HIPEC. Careful selection of the patients based on numerous patient and tumor factors is the need of the hour to achieve maximal benefit with CRS and HIPEC [5]. The role of CRS and HIPEC in PM of gastric and colorectal origin is being explored worldwide with encouraging results.

\section{Gastrointestinal Cancer-Related Peritoneal Metastasis}

There are two clinical scenarios in which PM is detected in a gastrointestinal cancer: i) synchronous and ii) metachronous. While synchronous PM warrants a radical surgical approach with CRS and HIPEC at presentation in a select group of patients to improve survival, the management of metachronous PM lies in its prevention. Identifying risk factors for metachronous PM at the first presentation and instituting prophylactic HIPEC in high-risk patients would help to prevent the occurrence of metachronous PM.

Why systemic chemotherapy fails to contain PM has always been a matter of debate. A number of hypotheses have been put forward to understand why the PM does not always respond to systemic chemotherapy: i) the presence of plasma-peritoneal barrier [6] limiting the access of intravenous chemotherapy to PM, ii) inadequate blood supply and oxygenation of the tumor cells coupled with their low apoptotic potential, and iii) poor tolerance of standard chemotherapeutic drugs such as cisplatin or irinotecan due to drug toxicity which results from the reduced metabolism and/or excretion (retention in ascites) of the drugs $[7,8]$.

However, in some patients, excluding low-grade mucinous spread, a response can be detected if staging and restaging include laparoscopy with visualization and biopsy of the lesions.

\section{Role of HIPEC in Gastrointestinal Cancer}

The role of HIPEC in the management of gastrointestinal cancer-related PM can be summarized as i) prophylactic to prevent metachronous PM in high-risk patients, ii) therapeutic to address synchronous PM after CRS, and iii) palliative to address intractable ascites in those patients who are otherwise not suitable for CRS.

The mechanism of action of HIPEC can be summarized as regional dose intensification at the level of the peritoneum without the associated systemic toxicity. The peritoneal-plasma barrier which restricts the movement of the systemic chemotherapeutic drug to reach the peritoneum helps during HIPEC to avoid absorption of the drug into the systemic circulation. High temperature of the intraperitoneal fluid selectively has a direct cell-killing effect on the tumor cells; furthermore, it potentiates tissue penetration and enhances the cytotoxic effect of the administered chemotherapeutic drugs [9].

\section{Gastric Cancer-Related Peritoneal Metastasis}

Gastric cancer is the fourth common cause of cancer-specific mortality in both males and females worldwide [10]. It is one of the most aggressive gastrointestinal cancers, with dismal 5-year survival rates of less than $25 \%$. Almost $40 \%$ of patients with gastric cancer have metastatic disease at presentation, and one third of them have PM. Interestingly, the peritoneum is the only site of metastases in one-tenth of these patients with metastatic disease [11]. Traditionally, these patients are treated with palliative chemotherapy and/or best supportive care and have a median survival of no more than 3-6 months and a 5-year survival of 0\% [7].

The addition of systemic chemotherapy marginally improved the median survival of the patients with metastatic gastric cancer to $8-12$ months $[12,13]$. Even addition of taxol and platinum group of drugs could not add a clinically meaningful rise in the median survival of patients with advanced gastric cancer [7]. When added to the conventional chemotherapy, the targeted agent trastuzumab could only make a difference of 2.5 months, though statistically significant, regarding the median survival (13.8 vs. 11.1 months) of these patients $[14,15]$. However, this was only the case for patients with HER2-positive gastric cancer.

The first report of CRS and HIPEC can be traced back to 1988 when Fujimoto et al. [16] published their experience of using CRS and HIPEC in 15 patients with advanced gastric cancer. They reported that all 9 of the 15 patients who had peritoneal seeding had complete resolution of ascites with an average follow-up of 7 months. A subsequent report by Fujimoto et al. [17] further strengthened the belief that aggressive surgery with HIPEC is a promising approach to address the PM in gastric cancer; they claimed to have achieved a 5 -year survival of $41.6 \%$ in a cohort of 18 patients with limited PM. Since then, there has been a series of publications which supported the role of CRS and HIPEC in gastric cancer-related PM.

There is another group of patients with gastric cancer which only has positive peritoneal cytology and does not have macroscopic peritoneal disease. Cancer cells exfoliating from the serosal surface of gastric tumors lead to this condition. However, various studies have proven that positive peritoneal cytology has as grave a prognosis as that of macroscopic peritoneal disease [5]. Intraperitoneal chemotherapy along with peritoneal lavage has been shown to be beneficial in reducing the subsequent peritoneal recurrence and in improving the survival. Further trials are eagerly awaited to provide high-quality evidence if HIPEC would be beneficial in this group of patients. 
Table 1. Ongoing phase III trials assessing the role of HIPEC in gastric cancer (https://clinicaltrials.gov/, as accessed on 01.07.2018)

\begin{tabular}{llll}
\hline S.No. & Study title & Study acronym & Location \\
\hline 1 & $\begin{array}{l}\text { Cytoreductive Surgery (CRS) With/Without } \\
\text { HIPEC in Gastric Cancer With Peritoneal } \\
\text { Carcinomatosis }\end{array}$ & GASTRIPEC & $\begin{array}{l}\text { Charité University, Berlin, multicentric study, } \\
\text { Germany }\end{array}$ \\
\hline 2 & $\begin{array}{l}\text { Efficacy of HIPEC in the Treatment of Locally } \\
\text { Advanced Gastric Cancer After radIcal } \\
\text { Gastrectomy With D2 }\end{array}$ & EHTLAGCRGD2 & multicentric study, China \\
\hline 3 & $\begin{array}{l}\text { Efficacy of HIPEC in the Treatment of Patients } \\
\text { With Locally Advanced Gastric Cancer }\end{array}$ & HIPEC & $\begin{array}{l}\text { Department of Abdominal Surgery (Section 2), } \\
\text { Affiliated Tumor Hospital of Guangzhou Medical } \\
\text { University, Guangzhou, Guangdong, China }\end{array}$ \\
\hline 4 & $\begin{array}{l}\text { D2 Resection and HIPEC (Hyperthermic } \\
\text { Intraperitoneal Chemoperfusion) in Locally } \\
\text { Advanced Gastric Carcinoma }\end{array}$ & GASTRICHIP & multicentric study, France and Spain \\
\hline 5 & $\begin{array}{l}\text { Radical Gastrectomy With/Without HIPEC in } \\
\text { Advanced Gastric Cancer Patients }\end{array}$ & none & Meng Wang, Nanjing, Jiangsu, China \\
\hline 6 & $\begin{array}{l}\text { Cytoreductive Surgery Combined With HIPEC } \\
\text { and Chemotherapy for Gastric Cancer With } \\
\text { Peritoneal Metastasis }\end{array}$ & none & multicentric study, China \\
\hline 7 & $\begin{array}{l}\text { Gastrectomy + Cytoreductive Surgery + HIPEC } \\
\text { for Gastric Cancer With Peritoneal Dissemination }\end{array}$ & PERISCOPE II & $\begin{array}{l}\text { Antoni van Leeuwenhoek/Netherlands Cancer } \\
\text { Institute, Amsterdam, the Netherlands }\end{array}$ \\
\hline
\end{tabular}

A meta-analysis of twenty prospective randomized controlled trials [18] including 2,145 patients with advanced gastric cancer generated a level I evidence for CRS and HIPEC as it revealed that 1 -year (odds ratio $(\mathrm{OR})=0.25,95 \%$ confidence interval $(\mathrm{CI})=$ $0.12-0.51)$, 2-year $(\mathrm{OR}=0.29,95 \% \mathrm{CI}=0.17-0.51)$, and 3 -year $(\mathrm{OR}=0.25,95 \% \mathrm{CI}=0.14-0.47)$ mortality were significantly better in patients with PM who underwent CRS and HIPEC compared to surgery alone. However, the difference in 5-year mortality between the patients with PM who had CRS and HIPEC compared to those with surgery alone failed to attain statistical significance $(\mathrm{OR}=$ $1.05,95 \% \mathrm{CI}=0.67-1.55)$. When elaborating on HIPEC, it would be imperative to highlight that HIPEC alone, i.e. with good-quality CRS to achieve a completeness-of-cytoreduction (CC) score of 0/1, would not be sufficient enough to enhance median survival. A meta-analysis of ten studies (441 treated patients with advanced gastric cancer), comprising one non-randomized prospective controlled trial, six prospective case series, and three retrospective case series, reported an overall median survival of 7.9 months; however, this median survival increased to 15 months for patients with CC scores of 0 or 1 compared to 3 months with best supportive care [19]. Sugarbaker's peritoneal cancer index (PCI) is an excellent method to assess the extent of PMs and correlates well with the likely CC score [20]. Thus, a selected group of patients with limited peritoneal disease $(\mathrm{PCI}<6)$ and without macronodular small bowel disease are ideal candidates for CRS and HIPEC. On the contrary, a PCI $>12$ should be considered a contraindication for CRS and HIPEC in gastric cancer. In a multi-institutional study to identify the prognostic factors for CRS and HIPEC, Glehen et al.
[21] highlighted that despite a complete cytoreduction, there were no long-term survivors ( $>3$ years) when the PCI was $>12$. Table 1 displays the ongoing phase III trials assessing the role of HIPEC in gastric cancer.

\section{Colorectal Cancer-Related Peritoneal Metastasis}

Although the liver is the most common site of metastasis in colorectal cancer, isolated PMs are seen in $15-25 \%$ of patients. Colorectal PM is the only gastrointestinal cancer (excluding appendiceal neoplasms) for which a plethora of evidence is available to support the role of CRS and HIPEC. Systemic chemotherapy has largely been a futile exercise in patients with colorectal cancer-associated PM, with a median survival of 12.7 months in patients with colorectal cancer and PM [22].

Although many uncontrolled studies indicated the benefit of CRS and HIPEC in patients with colorectal cancer and PM, the first randomized controlled trial that clearly highlighted the survival advantage of CRS and HIPEC over systemic chemotherapy was published by Verwaal et al. [4] in 2003. In a study of 205 patients, CRS and HIPEC led to a statistically significant survival benefit of almost 10 months (22.3 vs. 12.6 months, $\mathrm{p}=0.032$ ) compared to either standard treatment consisting of systemic chemotherapy (fluorouracil/leucovorin) with or without palliative surgery [4]. However, the authors cautioned that a complete cytoreduction is necessary to achieve optimal results with CRS and HIPEC. Verwaal et al. [23] published a follow-up study with a long median 
Table 2. Ongoing phase III trials assessing the role of HIPEC in colorectal cancer (data from https://clinicaltrials.gov/, as accessed on 01.07.2018)

\begin{tabular}{|c|c|c|c|}
\hline S.No. & Study title & Study acronym & Location \\
\hline 1 & $\begin{array}{l}\text { Adjuvant Hyperthermic Intraperitoneal } \\
\text { Chemotherapy (HIPEC) Versus no HIPEC in } \\
\text { Locally Advanced Colorectal Cancer }\end{array}$ & APEC & $\begin{array}{l}\text { Fudan University Shanghai Cancer Center, } \\
\text { Shanghai, China }\end{array}$ \\
\hline 2 & $\begin{array}{l}\text { Clinical Trial to Evaluate Safety and Efficacy of } \\
\text { Hyperthermic Intra-peritoneal Chemotherapy } \\
\text { (HIPEC) With Mitomycin C Used During Surgery } \\
\text { for Treatment of Locally Advanced Colorectal } \\
\text { Carcinoma }\end{array}$ & none & $\begin{array}{l}\text { Hospital Universitario Reina Sofía, Córdoba, } \\
\text { Spain }\end{array}$ \\
\hline 3 & $\begin{array}{l}\text { Trial Comparing Simple Follow-up to Exploratory } \\
\text { Laparotomy Plus 'in Principle' (Hyperthermic } \\
\text { Intraperitoneal Chemotherapy) HIPEC in } \\
\text { Colorectal Patients }\end{array}$ & ProphyloCHIP & Institut Gustave Roussy, Villejuif, France \\
\hline 4 & $\begin{array}{l}\text { Trial Evaluating Surgery With Hyperthermic } \\
\text { Intraperitoneal Chemotherapy (HIPEC) in } \\
\text { Treating Patients With a High Risk of Developing } \\
\text { Colorectal Peritoneal Carcinomatosis }\end{array}$ & none & multicentric study, China \\
\hline 5 & $\begin{array}{l}\text { Second and Third Look Laparoscopy in pT4 Colon } \\
\text { Cancer Patients for Early Detection of Peritoneal } \\
\text { Metastases }\end{array}$ & COLOPEC-II & $\begin{array}{l}\text { (a) Flevoziekenhuis, Almere, the Netherlands; } \\
\text { (b) Academic Medical Center, Amsterdam, } \\
\text { the Netherlands }\end{array}$ \\
\hline 6 & $\begin{array}{l}\text { Perioperative Systemic Therapy and Surgery } \\
\text { Versus Surgery Alone for Resectable Colorectal } \\
\text { Peritoneal Metastases }\end{array}$ & CAIRO6 & multicentric study, the Netherlands \\
\hline
\end{tabular}

follow-up of 46 months and reaffirmed the previous findings - the median survival was 21.8 months (95\% CI 19.0-25.5 months) and the 1-, 3-, and 5-year survival rates were 75,28 , and $19 \%$, respectively. The authors further elaborated that the median survival was only 5 months in patients who could not have complete cytoreduction (gross residual macroscopic tumor) compared to 42.9 and 17.4 months in patients who either had complete cytoreduction or minimal residual disease, respectively [23]. However, as the more effective platinum-based systemic chemotherapy came into practice, the role of CRS and HIPEC was questioned as the previous trials had used fluorouracil/leucovorin-based chemotherapy in the control arm. A French study revealed that systemic chemotherapy containing oxaliplatin or irinotecan alone could lead to a median survival of 23.9 months compared to 62.7 months when it was accompanied by CRS and HIPEC. The 2-year and 5-year overall survival rates were also better in the CRS and HIPEC group compared to systemic chemotherapy alone ( 81 vs. $65 \%$ and 51 vs. $13 \%$, respectively) [2]. Recently reported results of the phase III Prodige 7 trial at the annual conference of the American Society of Clinical Oncology further confirmed that CRS led to an impressive median survival of 41 months in those patients with colorectal cancer-related PM; however, the trial could not demonstrate the benefit of adding HIPEC to CRS [24].

The next challenge in the management of colorectal cancer-related PM is the identification of the variables which are likely to influence survival after CRS and HIPEC. A meta-analysis confirmed that concurrent liver metastasis, lymph node metastasis, higher Eastern Cooperative Oncology Group score, tumor differentiation, and signet ring cell histology are all negative prognostic variables regarding overall survival after CRS and HIPEC [25]. Table 2 displays the ongoing phase III trials assessing the role of HIPEC in colorectal cancer.

\section{Conclusion}

A multidisciplinary approach combining the benefits of both CRS and HIPEC, and systemic chemotherapy is the way forward to manage patients with gastrointestinal cancer and peritoneal carcinomatosis. A proper selection of the patients for CRS and HIPEC is necessary to achieve maximal oncological benefit and minimal perioperative morbidity and mortality.

\section{Disclosure Statement}

All authors have no conflict of interest. 


\section{References}

1 Roviello F, Caruso S, Marrelli D, et al: Treatment of peritoneal carcinomatosis with cytoreductive surgery and hyperthermic intraperitoneal chemotherapy: state of the art and future developments. Surg Oncol 2011; 20:e38-54.

2 Elias D, Lefevre JH, Chevalier J, et al: Complete cytoreductive surgery plus intraperitoneal chemohyperthermia with oxaliplatin for peritoneal carcinomatosis of colorectal origin. J Clin Oncol 2009;27:681-685.

3 Franko J, Ibrahim Z, Gusani NJ, Holtzman MP, Bartlett DL, Zeh HJ: Cytoreductive surgery and hyperthermic intraperitoneal chemoperfusion versus systemic chemotherapy alone for colorectal peritoneal carcinomatosis. Cancer 2010;116:3756-3762.

4 Verwaal VJ, van Ruth S, de Bree E, et al: Randomized trial of cytoreduction and hyperthermic intraperitoneal chemotherapy versus systemic chemotherapy and palliative surgery in patients with peritoneal carcinomatosis of colorectal cancer. J Clin Oncol 2003;21: 3737-3743.

5 Sugarbaker PH: Gastric cancer: prevention and treatment of peritoneal metastases. J Cancer Metastasis Treat 2018;4:7.

6 Jacquet P, Sugarbaker PH: Peritoneal-plasma barrier. Cancer Treat Res 1996;82:53-63.

7 Seshadri RA, Glehen O: Cytoreductive surgery and hyperthermic intraperitoneal chemotherapy in gastric cancer. World J Gastroenterol 2016;22:1114-1130.

8 Shirao K, Boku N, Yamada Y, et al: Randomized phase III study of 5-fluorouracil continuous infusion vs. sequential methotrexate and 5-fluorouracil therapy in far advanced gastric cancer with peritoneal metastasis (JCOG0106). Jpn J Clin Oncol 2013;43:972-980.

9 González-Moreno S, González-Bayón LA, Ortega-Pérez G: Hyperthermic intraperitoneal chemotherapy: rationale and technique. World J Gastrointest Oncol 2010;2:68-75.
10 Torre LA, Bray F, Siegel RL, Ferlay J, Lortet-Tieulent Jemal A: Global cancer statistics, 2012. CA Cancer J Clin 2015;65:87-108.

11 Thomassen I, van Gestel YR, van Ramshorst B, et al: Peritoneal carcinomatosis of gastric origin: a population-based study on incidence, survival and risk factors. Int J Cancer 2014;134:622-628.

12 Pyrhönen S, Kuitunen T, Nyandoto P, Kouri M: Randomised comparison of fluorouracil, epidoxorubicin and methotrexate (FEMTX) plus supportive care with supportive care alone in patients with non-resectable gastric cancer. Br J Cancer 1995;71:587-591.

13 Glimelius B, Ekström K, Hoffman K, et al: Randomized comparison between chemotherapy plus best supportive care with best supportive care in advanced gastric cancer. Ann Oncol 1997;8:163-168.

14 Bang Y-J, Van Cutsem E, Feyereislova A, et al: Trastuzumab in combination with chemotherapy versus chemotherapy alone for treatment of HER2-positive advanced gastric or gastro-oesophageal junction cancer (ToGA): a phase 3, open-label, randomised controlled trial. Lancet Lond Engl 2010;376:687-697.

15 Garg PK, Jain BK: New cancer drugs at the cost of bankruptcy: will the oncologist tell the patients the benefit in terms of days/weeks added to life? Oncologist 2014;19:1291-1291.

16 Fujimoto S, Shrestha RD, Kokubun M, et al: Intraperitoneal hyperthermic perfusion combined with surgery effective for gastric cancer patients with peritoneal seeding. Ann Surg 1988;208:36-41.

17 Fujimoto S, Takahashi M, Mutou T, et al: Improved mortality rate of gastric carcinoma patients with peritoneal carcinomatosis treated with intraperitoneal hyperthermic chemoperfusion combined with surgery. Cancer 1997;79:884-891
18 Coccolini F, Cotte E, Glehen O, et al: Intraperitoneal chemotherapy in advanced gastric cancer. Meta-analysis of randomized trials. Eur J Surg Oncol 2014;40:1226.

9 Gill RS, Al-Adra DP, Nagendran J, et al: Treatment of gastric cancer with peritoneal carcinomatosis by cytoreductive surgery and HIPEC: a systematic review of survival, mortality, and morbidity. J Surg Oncol 2011; 104:692-698.

20 Jacquet P, Sugarbaker PH: Clinical research methodologies in diagnosis and staging of patients with peritoneal carcinomatosis. Cancer Treat Res 1996;82:359374.

1 Glehen O, Gilly FN, Arvieux C, et al: Peritoneal carcinomatosis from gastric cancer: a multi-institutional study of 159 patients treated by cytoreductive surgery combined with perioperative intraperitoneal chemotherapy. Ann Surg Oncol 2010;17:2370-2377.

22 Franko J, Shi Q, Goldman CD, et al: Treatment of colorectal peritoneal carcinomatosis with systemic chemotherapy: a pooled analysis of North Central Cancer Treatment Group Phase III Trials N9741 and N9841. J Clin Oncol 2012;30:263-267.

23 Verwaal VJ, van Ruth S, Witkamp A, Boot H, van Slooten G, Zoetmulder FAN: Long-term survival of peritoneal carcinomatosis of colorectal origin. Ann Surg Oncol 2005; 12:65-71.

24 Quenet F, Elias D, Roca L, et al: A UNICANCER phase III trial of hyperthermic intra-peritoneal chemotherapy (HIPEC) for colorectal peritoneal carcinomatosis (PC): PRODIGE 7. J Clin Oncol 2018; 36(suppl):LBA3503-LBA3503.

25 Kwakman R, Schrama AM, van Olmen JP, et al: Clinicopathological parameters in patient selection for $\mathrm{cy}$ toreductive surgery and hyperthermic intraperitoneal chemotherapy for colorectal cancer metastases: a meta-analysis. Ann Surg 2016;263:1102-1111. 